\title{
Approximate solutions of some problems of scattering of surface water waves by vertical barriers
}

\author{
A CHOUDHARY® and S C MARTHA*D \\ Department of Mathematics, Indian Institute of Technology Ropar, Rupnagar 140001, India \\ e-mail: arunc@iitrpr.ac.in; scmartha@gmail.com \\ http://www.iitrpr.ac.in/arun-choudhary; https://sites.google.com/site/scmartha/
}

MS received 10 June 2016; revised 17 November 2016; accepted 20 November 2016

\begin{abstract}
A class of mixed boundary value problems (bvps), occurring in the study of scattering of surface water waves by thin vertical rigid barriers placed in water of finite depth, is examined for their approximate solutions. Two different placings of vertical barriers are analyzed, namely, (i) a partially immersed barrier and (ii) a bottom standing barrier. The solutions of the bvps are obtained by utilizing the eigenfunction expansion method, leading to a mathematical problem of solving over-determined systems of linear algebraic equations. The methods of analytical least-square approximation as well as algebraic least-square approximation are employed to solve the corresponding over-determined system of linear algebraic equations and thereby evaluate the physical quantities, namely, the reflection and transmission coefficients. Further, the absolute values of the reflection coefficients are compared to the known results obtained by utilizing a Galerkin type of approximate method after reducing the bvps to integral equations whose complete solutions are difficult to be determined. Various combinations of discretization of the resulting dual series relations obtained in the present analysis are employed to determine the least-square solution.
\end{abstract}

Keywords. Mixed boundary value problem; over-determined system; least-square approximation; reflection coefficient; transmission coefficient.

\section{Introduction}

The interaction of surface waves with a fixed or moving obstaclue has long standing interest in many engineering applications, e.g., breakwaters protecting harbour from the sea and wavemakers, etc. The theoretical studies of twodimensional reflection and transmission of surface waves were initially carried out by Dean [1] and then by Ursell [2] and others. The problems of solving the governing equations in the above study, together with specific boundary conditions, have been investigated by various authors in the literature. For instance, Ursell [2] employed the singular integral equation approach in conjunction with Havelock's expansion to obtain an analytical solution of the problem in deep water. Losada et al [3] solved a similar problem in uniform finite depth of water with the aid of the eigenfunction expansion method, whereas various other authors have attempted to compute approximate solutions. Mandal and Dolai [4], for example, studied the problem of Losada et al [3] and solved it using a single-term Galerkin approximation method and presented the upper and lower bounds for the reflection and transmission coefficients. The actual values of these coefficients have not been found in

*For correspondence their work and efforts have been made to obtain the actual mathematical values of the unknowns, in the present paper. Later, Mandal and Chakrabarti [5] employed the method of Galerkin approximation to determine approximate solutions of a number of water wave scattering problems involving thin vertical barriers in deep water as well as in water of finite depth. Sahoo et al [6] studied the trapping of surface waves by porous barrier in water of finite depth. They have used the eigenfunction expansion method to solve this problem. The interaction of laminar wakes with free-surface waves generated by a moving body beneath the surface of an incompressible fluid was solved by Lu [7] using the method of integral transforms. In 2011, Feng and Lu [8] analysed the problem of interaction of surface water waves with floating structures of arbitrary shapes and its solution was obtained with the aid of an eigenfunction expansion method. Recently in 2013, Chakrabarti and Mohapatra [9] obtained the solution of the problem involving the scattering of water waves by floating structures using an algebraic least-square as well as singular value decomposition approach.

In the present study, we consider oblique wave scattering by a rigid vertical barrier, placed in two different manners in water of finite depth (figure 1). The problems under consideration give rise to a class of important mixed boundary value problems (bvps) involving Laplace's 


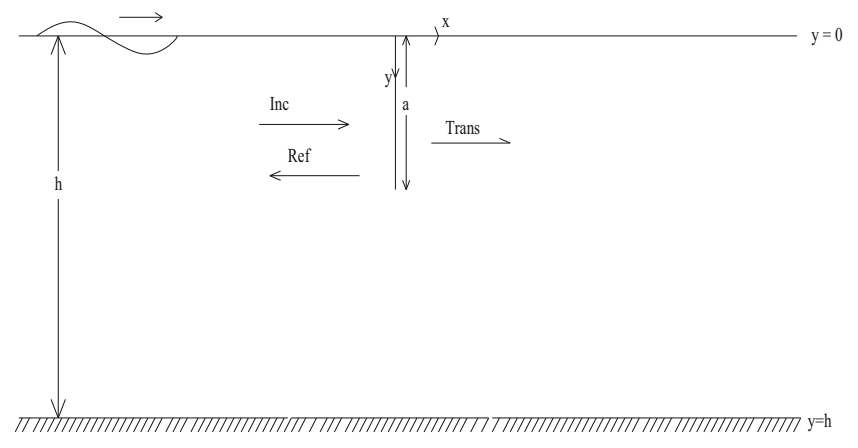

(a)

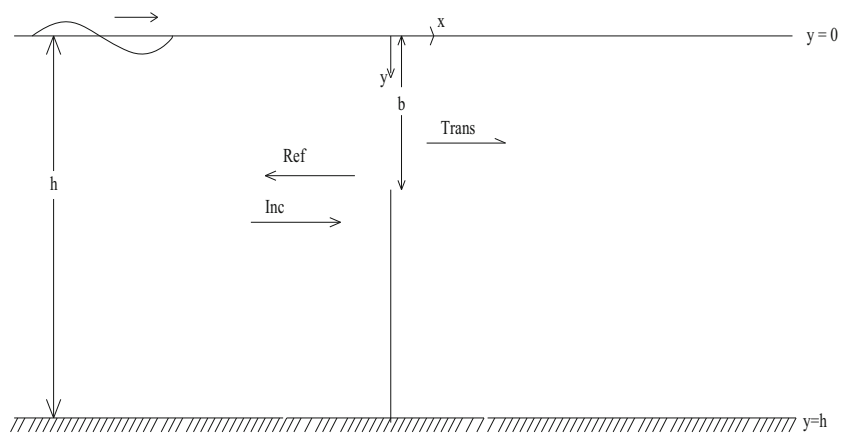

(b)

Figure 1. Definition sketch for two types of rigid barriers.

equation. Such problems deserve special attention for the determination of their complete solutions. To the best of our knowledge, no unified method exists for the most general problems of this class. Here, we have examined these problems for their complete approximate solutions. Our main intention in this paper is to employ a simple known technique from linear algebra to the problem at hand, in order to produce fast and effective numerical solutions, without following a traditional integral equation approach, which is more complicated.

The mixed boundary value problems are solved with the aid of an eigenfunction expansion method, leading to overdetermined systems of linear algebraic equations. It turns out that analytical least-square and algebraic least-square techniques can be employed to produce best solutions of such over-determined systems of equations. We found that these techniques yield numerical values of the reflection and transmission coefficients with good accuracy. Further, both uniform and non-uniform discretizations of the domain of the problem are considered while implementing the algebraic least-square method.

\section{Formulation of the problem}

Scattering of oblique waves by vertical barrier is analysed under the assumption of small-amplitude water wave theory in water of uniform finite depth. The problem is analysed in a three dimensional Cartesian co-ordinate system $(x, y, z)$ where the $x z$-plane represents the undisturbed free surface of the fluid and the positive $y$-axis is directed vertically downward. Let a thin vertical barrier denoted by $L$ be fixed in the fluid whose position (figure 1) may be described as follows:

(a) Case-I: $x=0,0 \leq y \leq a$ (a partially immersed barrier), (b) Case-II: $x=0, b \leq y \leq h$ (a bottom standing barrier).

The fluid is assumed to be incompressible and inviscid, and the flow is irrotational under the action of gravity and simple harmonic in time. The waves under this study are actually the waves governed by the potential giving rise to changing amplitude in the $y$-direction and the waves are assumed to be propagate in the $x z$-plane, producing the potential of the form $f(y) \mathrm{e}^{\mathrm{i}(\mu x+v z-\sigma t)}$. A progressive wave represented by the velocity potential $\Phi_{\text {inc }}=$ $\operatorname{Re}\left\{\hat{\phi}(x, y) \mathrm{e}^{\mathrm{i}(v z-\sigma t)}\right\}$ is incident obliquely upon the vertical barrier, where $\hat{\phi}(x, y)=\left(\mathrm{e}^{\mathrm{i} \mu x} \cosh \hat{k_{0}}(h-y)\right) / \cosh \hat{k_{0}} h$, $\mu=\hat{k_{0}} \cos \theta, v=\hat{k_{0}} \sin \theta, \theta$ being the angle of incidence, $K=\sigma^{2} / g, \sigma$ is the angular frequency of the incoming water wave train with time dependence $\mathrm{e}^{-\mathrm{i} \sigma t}$ and $g$ is the acceleration due to gravity. Here, $k=\hat{k_{0}}$ is the wave number of the incident wave, the positive real root of the transcendental equation $K-k \tanh k h=0$.

Uniformity in the $z$-direction helps in expressing the velocity potential in separable form $\psi(z, t) \phi(x, y)$; using the periodicity in time gives rise to the representation of the velocity potential as $\operatorname{Re}\left\{\phi(x, y) \mathrm{e}^{\mathrm{i}(v z-\sigma t)}\right\}$.

Assuming linear theory, $\phi(x, y)$ satisfies the bvp as given by

$$
\frac{\partial^{2} \phi}{\partial x^{2}}+\frac{\partial^{2} \phi}{\partial y^{2}}-v^{2} \phi=0, \quad \text { in the fluid region, }
$$

$$
\frac{\partial \phi}{\partial y}+K \phi=0, \quad \text { on } y=0,
$$

$$
\frac{\partial \phi}{\partial y}=0, \quad \text { on } y=h,
$$

$$
\left.\frac{\partial \phi}{\partial x}\right|_{x=0^{-}}=\left.\frac{\partial \phi}{\partial x}\right|_{x=0^{+}}=0, \quad \text { on } x=0, y \in L,
$$

$$
\begin{gathered}
\left.\frac{\partial \phi}{\partial x}\right|_{x=0^{-}}=\left.\frac{\partial \phi}{\partial x}\right|_{x=0^{+}}, \quad \text { on } x=0, y \in \bar{L}, \\
\left.\phi\right|_{x=0^{-}}=\left.\phi\right|_{x=0^{+}}, \quad \text { on } x=0, y \in \bar{L}, \\
\phi(x, y) \rightarrow\left\{\begin{array}{cc}
\hat{\phi}(x, y)+R \hat{\phi}(-x, y) & \text { as } x \rightarrow-\infty, \\
T \hat{\phi}(x, y) & \text { as } x \rightarrow \infty,
\end{array}\right.
\end{gathered}
$$


where $\bar{L}$ represents the gap so that $L \bigcup \bar{L}$ is $[0, h] ; R$ and $T$ are unknown complex constants associated with the amplitude of the reflected and transmitted waves, respectively. The relation (4) is due to the horizontal velocity being zero along the rigid wall of the barrier. The relations (5) and (6) are due to the fact that the horizontal velocity and pressure are continuous across the gap. The derivation of relation (6) is given in "Appendix". The effect of surface tension is assumed to be negligible for the present problem. The unknown constants $R$ and $T$ are to be determined, along with the unknown potential function $\phi(x, y)$, by solving the bvps, satisfying the governing equation (1) and conditions (2)-(7).

\section{Methods of solution}

The velocity potential $\phi(x, y)$ satisfying relation (1) along with the boundary conditions (2), (3) and (7) can be expressed in the following forms of eigenfunction expansion:

$$
\phi(x, y)= \begin{cases}T \hat{\phi}(x, y)+\sum_{n=1}^{\infty} A_{n} \cos k_{n}(h-y) \mathrm{e}^{-s_{n} x} & \text { for } x>0, \\ \hat{\phi}(x, y)+R \hat{\phi}(-x, y)+\sum_{n=1}^{\infty} B_{n} \cos k_{n}(h-y) \mathrm{e}^{s_{n} x} & \text { for } x<0,\end{cases}
$$

where $s_{n}=\sqrt{k_{n}^{2}+v^{2}}$ and $k_{n}(n=1,2, \ldots)$ are the positive real roots of the transcendental equation $K+k \tan k h=0$. Here $R, T, A_{n}$ and $B_{n}$ are unknown complex constants to be determined to obtain the velocity potential $\phi(x, y)$ completely. It is noted that the eigenfunctions $\cos k_{n}(h-$ $y), n=0,1,2, \ldots$ form a complete orthogonal set with

$$
\frac{1}{h} \int_{0}^{h} \cos k_{n}(h-y) \cos k_{m}(h-y) d y=\delta_{m n} N\left(k_{n}\right),
$$

where $N\left(k_{n}\right)=\left(2 k_{n} h+\sin 2 k_{n} h\right) /\left(4 k_{n} h\right)$ with $k_{0}=-\mathrm{i} \hat{k_{0}}$ and $\delta_{m n}$ is the Kronecker delta. In relation (8), the sum represents the non-propagating modes, which decay rapidly away from the barrier, and the other terms represent the propagating mode.

The mixed bvps in this configuration lead to integral equations of particular type as shown by previous workers $[4,5]$, whose complete solutions are difficult to determine. Instead, we have reduced the problem under consideration into a set of dual series relations as given in (11) and (12), which are finally solved approximately.

In the next section, we present approximate solutions of these dual series relations by the analytical and algebraic leastsquare methods. For the purpose of utilizing the algebraic least-square method, we have used discretized points in the dual series relations as (i) equally spaced points and (ii) unequally spaced points, after converting the respective interval into $(-1,1)$ using standard transformations. Numerical results are obtained and are listed in tabular forms.

\subsection{Solution by analytical least-square method}

Using the fact that the condition $\partial \phi /\left.\partial x\right|_{x=0^{-}}=\partial \phi /\left.\partial x\right|_{x=0^{+}}$ holds good for all $y(0<y<h)$ and using Havelock's inversion formula (see Ursell [2]), we find that

$$
R+T=1 \text { and } A_{n}=-B_{n} .
$$

Using the relation (8) in relations (4) and (6), we get

$$
\begin{gathered}
\sum_{n=0}^{\infty} B_{n} s_{n} h \cos k_{n}(h-y)=0, \quad \text { on } x=0, y \in L, \\
\frac{\cosh \hat{k_{0}}(h-y)}{\cosh \hat{k_{0}} h}+\sum_{n=0}^{\infty} B_{n} \cos k_{n}(h-y)=0, \quad \text { on } x=0, y \in \bar{L} .
\end{gathered}
$$

where

$$
s_{0}=-\mathrm{i} \mu \text { and } B_{0}=\frac{R-1}{\cosh \hat{k_{0} h}} .
$$

The relations (11) and (12) can be combined to form one relation, as given by

$$
G(y)=0,0<y<h,
$$

where

$$
G(y)= \begin{cases}\sum_{n=0}^{\infty} B_{n} s_{n} h \cos k_{n}(h-y) & \text { on } y \in L, \\ \frac{\cosh \hat{k_{0}}(h-y)}{\cosh \hat{k_{0}} h}+\sum_{n=0}^{\infty} B_{n} \cos k_{n}(h-y) & \text { on } y \in \bar{L} .\end{cases}
$$

For the determination of the unknowns $B_{n}$, the method of analytical least-square approximation can be used, by minimizing the error as given by the following formula:

Error $=\left(\int_{o}^{h}|G(y)|^{2} \mathrm{~d} y\right)^{1 / 2}=\left(\int_{y \in \bar{L}}|G(y)|^{2} \mathrm{~d} y+\int_{y \in L}|G(y)|^{2} \mathrm{~d} y\right)^{1 / 2}$.

Minimizing the above error with respect to $B_{n}$ gives rise to the following normal equations:

$$
\int_{y \in \bar{L}} G^{*}(y) \frac{\partial G(y)}{\partial B_{m}} \mathrm{~d} y+\int_{y \in L} G^{*}(y) \frac{\partial G(y)}{\partial B_{m}} \mathrm{~d} y=0, m=0,1,2, \ldots
$$

where $G^{*}(y)$ is the complex conjugate of $G(y)$.

On substituting the value of $G^{*}$ and the derivatives of $G$ with respect to the unknowns, relation (16) gives a system of equations as given by 


$$
\begin{aligned}
& \sum_{n=0}^{\infty} B_{n}^{*}\left[\frac{1}{h} \int_{y \in \bar{L}} \cos k_{n}(h-y) \cos k_{m}(h-y) \mathrm{d} y\right. \\
& \left.+\left(s_{m} h\right)\left(s_{n}^{*} h\right) \frac{1}{h} \int_{y \in L} \cos k_{n}(h-y) \cos k_{m}(h-y) \mathrm{d} y\right] \\
& \quad=-\frac{1}{h} \int_{y \in \bar{L}} \frac{\cosh \hat{k_{0}}(h-y) \cos k_{m}(h-y)}{\cosh \hat{k_{0}} h} \mathrm{~d} y, m=0,1,2, \ldots
\end{aligned}
$$

For each type of barrier configuration, the limits of integrals get changed and relation (17) produces a determined system of linear equations involving the unknowns $B_{n}(n=$ $0,1, \ldots, N)$ after truncating $m, n$ to a finite number $N$. This determined system is then solved by the standard method, yielding the numerical values of $B_{n}$ as well as the numerical values of $R$ using relation (13).

\subsection{Solution by algebraic least-square method}

The relations (11) and (12) can be treated as dual series relations [10]. The solution of these dual series relations can be obtained approximately by applying the method of algebraic least squares, which yields the value of unknowns $B_{n}(n=0,1,2, \ldots)$ and hence the reflection coefficient $R$. To apply this type of algebraic least-square method, we assume $y_{1}, y_{2}, \ldots$ in $L$, and $\hat{y_{1}}, \hat{y_{2}}, \ldots$ in $\bar{L}$, to be the infinite number of discretized points in relations (11) and (12), respectively, which lead to an over-determined system with infinite number of equations and finite unknowns, given by

$$
A X=b
$$

where

$$
\begin{aligned}
& A=\left[\begin{array}{cccc}
s_{0} h \cos k_{0} h\left(1-y_{1} / h\right) & s_{1} h \cos k_{1} h\left(1-y_{1} / h\right) & s_{2} h \cos k_{2} h\left(1-y_{1} / h\right) & \ldots \\
\cos k_{0} h\left(1-\hat{y_{1}} / h\right) & \cos k_{1} h\left(1-\hat{y_{1}} / h\right) & \cos k_{2} h\left(1-\hat{y_{1}} / h\right) & \ldots \\
s_{0} h \cos k_{0} h\left(1-y_{2} / h\right) & s_{1} h \cos k_{1} h\left(1-y_{2} / h\right) & s_{2} h \cos k_{2} h\left(1-y_{2} / h\right) & \ldots \\
\cos k_{0} h\left(1-\hat{y_{2}} / h\right) & \cos k_{1} h\left(1-\hat{y_{2}} / h\right) & \cos k_{2} h\left(1-\hat{y_{2}} / h\right) & \ldots \\
\vdots & \vdots & \vdots & \ddots
\end{array}\right] \\
& X=\left[\begin{array}{c}
B_{0} \\
B_{1} \\
B_{2} \\
\vdots
\end{array}\right], b=\left[\begin{array}{c}
0 \\
-\frac{\cosh \hat{k_{0}} h\left(1-\hat{y_{1}} / h\right)}{\cosh \hat{k_{0}} h} \\
0 \\
-\frac{\cosh \hat{k_{0}} h\left(1-\hat{y_{2}} / h\right)}{\cosh \hat{k_{0}} h} \\
\vdots
\end{array}\right]
\end{aligned}
$$

Choosing $\hat{m}_{1}$ number of points in $L$ and $\hat{m}_{2}$ number of points in $\bar{L}$, we have $\hat{m}_{1}+\hat{m}_{2}=\hat{m}$ (say) number of equations. Taking $N$ number of unknowns with $\hat{m}>N$, the leastsquare solution is found for which the following normal system has to be solved:

$$
A^{*} A X=A^{*} b,
$$

where $A^{*}$ denotes the conjugate transpose of $A$.

If $A$ has linearly independent columns, then the leastsquare solution is unique, as given by

$$
X=\left(A^{*} A\right)^{-1} A^{*} b
$$

It may be noted to be able to utilize the algebraic approach described here successfully, the occurrence of ill-conditioned matrixes must be avoided. This problem can be avoided by choosing appropriate points of discretization and parameters as described in the next section.

For the two types of barrier configurations, the corresponding over-determined system of linear equations (18) is to be solved to find the numerical values of the unknowns $X=\left[B_{0}, B_{1}, \ldots, B_{N}\right]^{T}$, for which the error is given by

$$
E=\|A X-b\|
$$

where II.II is the $L_{2}$ norm.

\section{Numerical results and discussion}

In this section, a MATLAB program is developed to determine the numerical values of the unknowns $B_{0}, B_{1}, \ldots, B_{N}$ from relation (17) by utilizing the analytical least-square approximation and also from relation (20) by employing algebraic least-square approximations. Further, from the values of $B_{0}$ the values of the reflection coefficient $R$ (using relation (13)) and its absolute value $|R|$ are obtained and compared with the lower bound (denoted by $R_{1}$ ) and upper bound (denoted by $R_{2}$ ) of $|R|$ given in [4] for different barriers (tables 1-4, 6-13).

\subsection{Equally spaced discretized points}

For applying the algebraic least-square method, in case of partially immersed barrier $(L=(0, a / h), \bar{L}=(a / h, 1)$ in non-dimensional form), the points $y_{i} / h=y_{1} / h+(i-1) h_{1}$, $i=1,2, \ldots, \hat{m}_{1}$ with $y_{1} / h=0, y_{\hat{m}_{1}} / h=a / h$ with spacing $h_{1}=(a / h) /\left(\hat{m}_{1}-1\right)$ are chosen in $L=(0, a / h)$, and the points $\quad \hat{y}_{i} / h=\hat{y}_{1} / h+(i-1) h_{2}, \quad i=1,2, \ldots, \hat{m}_{2} \quad$ with $\hat{y}_{1} / h=a / h, \quad \hat{y}_{\hat{m}_{2}} / h=1$ with spacing $h_{2}=(1-a / h) /$ $\left(\hat{m}_{2}-1\right)$, are chosen in $\bar{L}=(a / h, 1)$. Similar discritized points are chosen in the case of bottom standing barrier. The number of unknowns $(N)$ taken in both the methods (methods of analytical least squares and algebraic least squares) are the same.

From tables $1-4$, it is observed that the absolute values of the reflection coefficient $|R|$, obtained by analytical leastsquare approximation and algebraic least-square approximation, are almost the same with the lower and upper bounds of $|R|$ given in [4].

For the case of partially immersed barrier, selected values of the unknowns $B_{1}, \ldots, B_{N}$ corresponding to non- 
Table 1. Reflection coefficient with $K h=0.2$ and $\theta=0$ for partially immersed barrier.

\begin{tabular}{|c|c|c|c|c|c|c|c|}
\hline \multirow{2}{*}{$a / h$} & \multicolumn{3}{|c|}{ Algebraic least squares } & \multicolumn{2}{|c|}{ Analytical least squares } & \multicolumn{2}{|c|}{ Mandal and Doali } \\
\hline & $\left(\hat{m_{1}}, \hat{m_{2}}, N\right)$ & $R$ & $|R|$ & $R$ & $|R|$ & $R_{1}$ & $R_{2}$ \\
\hline \multirow[t]{3}{*}{0.2} & $(50,50,70)$ & $0.0003-0.0174 \mathrm{i}$ & 0.0174 & $0.0004-0.0184 \mathrm{i}$ & 0.0184 & & \\
\hline & $(75,75,100)$ & $0.0003-0.0173 \mathrm{i}$ & 0.0173 & $0.0003-0.0180 \mathrm{i}$ & 0.0198 & 0.0176 & 0.0176 \\
\hline & $(100,100,120)$ & $0.0003-0.0176 \mathrm{i}$ & 0.0176 & $0.0003-0.0179 \mathrm{i}$ & 0.0184 & & \\
\hline \multirow[t]{3}{*}{0.4} & $(50,50,90)$ & $0.0052-0.0715 \mathrm{i}$ & 0.0717 & $0.0055-0.0735 \mathrm{i}$ & 0.0737 & & \\
\hline & $(75,75,137)$ & $0.0051-0.0711 \mathrm{i}$ & 0.0713 & $0.0053-0.0727 \mathrm{i}$ & 0.0728 & 0.0712 & 0.0713 \\
\hline & $(100,100,180)$ & $0.0051-0.0711 \mathrm{i}$ & 0.0713 & $0.0053-0.0722 \mathrm{i}$ & 0.0724 & & \\
\hline \multirow[t]{3}{*}{0.6} & $(50,50,85)$ & $0.0311-0.1723 \mathrm{i}$ & 0.1760 & $0.0322-0.1762 \mathrm{i}$ & 0.1791 & & \\
\hline & $(75,75,125)$ & $0.0308-0.1726 \mathrm{i}$ & 0.1754 & $0.0317-0.1749 \mathrm{i}$ & 0.1777 & 0.1740 & 0.1740 \\
\hline & $(100,100,170)$ & $0.0306-0.1723 \mathrm{i}$ & 0.1750 & $0.0313-0.1741 \mathrm{i}$ & 0.1769 & & \\
\hline
\end{tabular}

Table 2. Reflection coefficient with $K h=0.2$ and $\theta=\pi / 6$ for partially immersed barrier.

\begin{tabular}{|c|c|c|c|c|c|c|c|}
\hline \multirow{2}{*}{$a / h$} & \multicolumn{3}{|c|}{ Algebraic least squares } & \multicolumn{2}{|c|}{ Analytical least squares } & \multicolumn{2}{|c|}{ Mandal and Doali } \\
\hline & $\left(\hat{m_{1}}, \hat{m_{2}}, N\right)$ & $R$ & $|R|$ & $R$ & $|R|$ & $R_{1}$ & $R_{2}$ \\
\hline \multirow{4}{*}{0.2} & $(50,50,70)$ & $0.0002-0.0151 \mathrm{i}$ & 0.0151 & $0.0003-0.0159 \mathrm{i}$ & 0.0159 & \multirow{4}{*}{0.0152} & \multirow{4}{*}{0.0152} \\
\hline & $(75,75,100)$ & $0.0002-0.0150 \mathrm{i}$ & 0.0150 & $0.0003-0.0156 \mathrm{i}$ & 0.0156 & & \\
\hline & $(100,100,125)$ & $0.0002-0.0152 \mathrm{i}$ & 0.0152 & $0.0002-0.0154 \mathrm{i}$ & 0.0154 & & \\
\hline & $(50,50,93)$ & $0.0038-0.0614 \mathrm{i}$ & 0.0615 & $0.0041-0.0635 \mathrm{i}$ & 0.0637 & & \\
\hline \multirow[t]{3}{*}{0.4} & $(75,75,135)$ & $0.0038-0.0617 \mathrm{i}$ & 0.0618 & $0.0040-0.0629 \mathrm{i}$ & 0.0630 & \multirow[t]{3}{*}{0.0616} & \multirow[t]{3}{*}{0.0617} \\
\hline & $(100,100,180)$ & $0.0038-0.0616 \mathrm{i}$ & 0.0617 & $0.0039-0.0625 \mathrm{i}$ & 0.0627 & & \\
\hline & $(50,50,85)$ & $0.0234-0.1509 \mathrm{i}$ & 0.1527 & $0.0242-0.1535 \mathrm{i}$ & 0.1554 & & \\
\hline \multirow[t]{2}{*}{0.6} & $(75,75,129)$ & $0.0231-0.15036 \mathrm{i}$ & 0.1520 & $0.0238-0.1522 \mathrm{i}$ & 0.1541 & \multirow[t]{2}{*}{0.1509} & \multirow[t]{2}{*}{0.1509} \\
\hline & $(100,100,172)$ & $0.0226-0.1486 \mathrm{i}$ & 0.1503 & $0.0236-0.1516 \mathrm{i}$ & 0.1534 & & \\
\hline
\end{tabular}

Table 3. Reflection coefficient with $K h=0.2$ and $\theta=0$ for bottom standing barrier.

\begin{tabular}{|c|c|c|c|c|c|c|c|}
\hline \multirow{2}{*}{$b / h$} & \multicolumn{3}{|c|}{ Algebraic least squares } & \multicolumn{2}{|c|}{ Analytical least squares } & \multicolumn{2}{|c|}{ Mandal and Doali } \\
\hline & $\left(\hat{m_{1}}, \hat{m_{2}}, N\right)$ & $R$ & $|R|$ & $R$ & $|R|$ & $R_{1}$ & $R_{2}$ \\
\hline \multirow[t]{3}{*}{0.2} & $(50,50,62)$ & $0.0927-0.2895 \mathrm{i}$ & 0.3039 & $0.0958+0.2933 \mathrm{i}$ & 0.3086 & & \\
\hline & $(75,75,98)$ & $0.0906-0.2869 \mathrm{i}$ & 0.3008 & $0.0934+0.2906 \mathrm{i}$ & 0.3053 & 0.2914 & 0.2923 \\
\hline & $(100,100,130)$ & $0.0901-0.2863 \mathrm{i}$ & 0.3002 & $0.0924+0.2893 \mathrm{i}$ & 0.3037 & & \\
\hline \multirow[t]{3}{*}{0.4} & $(50,50,80)$ & $0.0206-0.1419 \mathrm{i}$ & 0.1434 & $0.0215+0.1446 \mathrm{i}$ & 0.1462 & & \\
\hline & $(75,75,130)$ & $0.0203-0.1411 \mathrm{i}$ & 0.1425 & $0.0210+0.1432 \mathrm{i}$ & 0.1447 & 0.1397 & 0.1397 \\
\hline & $(100,100,175)$ & $0.0203-0.1409 \mathrm{i}$ & 0.1424 & $0.0208+0.1425 \mathrm{i}$ & 0.1440 & & \\
\hline \multirow[t]{3}{*}{0.6} & $(50,50,92)$ & $0.0034-0.0575 \mathrm{i}$ & 0.0579 & $0.0036+0.0596 \mathrm{i}$ & 0.0597 & & \\
\hline & $(75,75,135)$ & $0.0034-0.0577 \mathrm{i}$ & 0.0578 & $0.0035+0.0590 \mathrm{i}$ & 0.0592 & 0.0573 & 0.0573 \\
\hline & $(100,100,175)$ & $0.0034-0.0579 \mathrm{i}$ & 0.0580 & $0.0035+0.0587 \mathrm{i}$ & 0.0588 & & \\
\hline
\end{tabular}

propagating modes from relation (20) are presented in table 5 .

\subsection{Unequally spaced discretized points}

Here, we consider the case of partially immersed barrier ( $L=(0, a / h), \bar{L}=(a / h, 1)$ in non-dimensional form) and use unequal discretization. The discretized points are chosen in such a manner that the step sizes in the interval $(0, a)$ $h)$ as well as in $(a / h, 1)$ are not equal. The transformation $y / h=\frac{a / h}{2}(t+1)$ changes the interval $(0, a / h)$ to $(-1,1)$ and the transformation $y / h=\left(\frac{1-a / h}{2}\right) t+\left(\frac{1+a / h}{2}\right)$ changes the interval $(a / h, 1)$ to $(-1,1)$.

Using these transformations the relations (11) and (12), respectively, can be rewritten as

$$
\sum_{n=0}^{\infty} B_{n} s_{n} h \cos k_{n} h\left(1-\frac{a / h}{2}(t+1)\right)=0 \text { on } \mathrm{t} \in(-1,1),
$$


Table 4. Reflection coefficient with $K h=0.2$ and $\theta=\pi / 6$ for bottom standing barrier.

\begin{tabular}{|c|c|c|c|c|c|c|c|}
\hline \multirow{2}{*}{$b / h$} & \multicolumn{3}{|c|}{ Algebraic least squares } & \multicolumn{2}{|c|}{ Analytical least squares } & \multicolumn{2}{|c|}{ Mandal and Doali } \\
\hline & $\left(\hat{m_{1}}, \hat{m_{2}}, N\right)$ & $R$ & $|R|$ & $R$ & $|R|$ & $R_{1}$ & $R_{2}$ \\
\hline \multirow[t]{3}{*}{0.2} & $(50,50,65)$ & $0.0705-0.2557 \mathrm{i}$ & 0.2652 & $0.0732+0.2596 \mathrm{i}$ & 0.2697 & & \\
\hline & $(75,75,95)$ & $0.0700-0.2549 \mathrm{i}$ & 0.26448 & $0.0760+0.2574 \mathrm{i}$ & 0.2672 & 0.2545 & 0.2553 \\
\hline & $(100,100,130)$ & $0.0689-0.2532 \mathrm{i}$ & 0.2624 & $0.0707+0.2560 \mathrm{i}$ & 0.2656 & & \\
\hline \multirow[t]{3}{*}{0.4} & $(50,50,90)$ & $0.0155-0.1235 \mathrm{i}$ & 0.1244 & $0.0160+0.1253 i$ & 0.1263 & & \\
\hline & $(75,75,130)$ & $0.0153-0.1225 \mathrm{i}$ & 0.1234 & $0.0158+0.1244 \mathrm{i}$ & 0.1254 & 0.1209 & 0.1209 \\
\hline & $(100,100,175)$ & $0.0152-0.1224 \mathrm{i}$ & 0.1233 & $0.0156+0.1238 \mathrm{i}$ & 0.1248 & & \\
\hline \multirow[t]{3}{*}{0.6} & $(50,50,80)$ & $0.0026-0.0510 \mathrm{i}$ & 0.0510 & $0.0027+0.0518 \mathrm{i}$ & 0.0519 & & \\
\hline & $(75,75,120)$ & $0.0026-0.0506 \mathrm{i}$ & 0.0507 & $0.0026+0.0512 \mathrm{i}$ & 0.0513 & 0.0495 & 0.0495 \\
\hline & $(100,100,150)$ & $0.0026-0.0505 \mathrm{i}$ & 0.0506 & $0.0026+0.0510 \mathrm{i}$ & 0.0510 & & \\
\hline
\end{tabular}

$$
\begin{aligned}
\sum_{n=0}^{\infty} & B_{n} \cos k_{n} h\left(1-\frac{1-a / h}{2} t-\frac{1+a / h}{2}\right) \\
= & \frac{-1}{\cosh \hat{k_{0}} h} \cosh \hat{k_{0}} h\left(1-\frac{1-a / h}{2} t-\frac{1+a / h}{2}\right) \\
& \text { on } \mathrm{t} \in(-1,1) .
\end{aligned}
$$

Similarly, the case of bottom standing barrier ( $L=(b / h, 1), \bar{L}=(0, b / h)$ in non-dimensional form) is handled using unequal discretization. We have chosen the discretized points in such a manner that the step sizes in the interval $(0, b / h)$ as well as in $(b / h, 1)$ are not equal. Here, the transformation $y / h=\frac{b / h}{2}(t+1)$ changes the interval $(0, b / h)$ to $(-1,1)$ and the transformation $y / h=\left(\frac{1-b / h}{2}\right) t+$ $\left(\frac{1+b / h}{2}\right)$ changes the interval $(b / h, 1)$ to $(-1,1)$. With these transformations the relations (11) and (12), respectively, can be rewritten as

$$
\begin{aligned}
& \sum_{n=0}^{\infty} B_{n} s_{n} h \cos k_{n} h\left(1-\frac{1-b / h}{2} t-\frac{1+b / h}{2}\right) \\
& =0 \text { on } \mathrm{t} \in(-1,1), \\
& \sum_{n=0}^{\infty} B_{n} \cos k_{n} h\left(1-\frac{b / h}{2}(t+1)\right) \\
& =\frac{-1}{\cosh \hat{k_{0}} h} \cosh \hat{k_{0}} h\left(1-\frac{b / h}{2}(t+1)\right) \\
& \quad \text { on } \mathrm{t} \in(-1,1) .
\end{aligned}
$$

\section{Type-I}

Let us choose the discretized points as $t_{i}=$ $\cos \left(\frac{(2 i-1) \pi}{2 \hat{m}_{1}}\right), i=1,2, \ldots, \hat{m}_{1}$, which are the zeros of Chebyshev's polynomial of the first kind (CPF), in relations (22) and (23) for the case of partially immersed barrier ((24) and (25) for the case of bottom standing barrier). Here, $\hat{m}_{1}=\hat{m}_{2}$. Then these relations lead to an over-determined systems of equations, which are solved with the aid of algebraic least-square method described in section
3.2, yielding the unknowns $B_{n}$. Using the values $B_{0}$, values of $R$ and hence $|R|$ are determined and compared to the known results [4] in tables 6 and 7.

\section{Type-II}

Choosing the discretized points $t_{i}=\cos \left(\frac{i \pi}{\hat{m}_{1}+1}\right), i=$ $1,2, \ldots, \hat{m}_{1}$, to be the zeros of Chebyshev's polynomial of the second kind (CPS) in relations (22) and (23) for the case of partially immersed barrier ((24) and (25) for the case of bottom standing barrier) and applying the method described in section 3.2, the values of the unknowns are obtained and hence the values of $R$ (tables 8 and 9). Here, we have also considered $\hat{m}_{1}=\hat{m}_{2}$.

\section{Type-III}

In this type, the discretized points $t_{i}=\cos \left(\frac{(2 i-1) \pi}{2 \hat{m}_{1}}\right), i=$ $1,2, \ldots, \hat{m}_{1}$, are the zeros of Chebyshev's polynomial of the first kind (CPF), which are used in relation (22) (in relation (24) for bottom standing barrier) and $t_{j}=\cos \left(\frac{j \pi}{\hat{m}_{2}+1}\right), j=$ $1,2, \ldots, \hat{m}_{2}$, the zeros of Chebyshev's polynomial of the second kind (CPS), are used in relation (23) (in relation (25) for bottom standing barrier). The values of $R$ are determined by algebraic least-square method and are shown in tables 10 and 11.

\section{Type-IV}

Here, the discretized points $t_{i}=\cos \left(\frac{i \pi}{\hat{m}_{1}+1}\right), i=$ $1,2, \ldots, \hat{m}_{1}$, are the zeros of Chebyshev's polynomial of the second kind used in relation (22) (in relation (24) for bottom standing barrier) and $t_{j}=\cos \left(\frac{(2 j-1) \pi}{2 \hat{m}_{2}}\right), j=1,2, \ldots, \hat{m}_{2}$ are the zeros of Chebyshev's polynomial of the first kind used in relation (23) (in relation (25) for bottom standing barrier). The values of the unknowns and hence the values of $R$ are presented in tables 12 and 13 .

Tables 6-13 for the case of unequal discretization show that the absolute values of the reflection coefficient $|R|$ obtained by algebraic least-square method are almost equal with the lower and upper bounds of $|R|$ given in [4].

The error (relation (21)) is computed for different values of $N$ and listed in table 14 for fixed values of $K h=$ 
Table 5. Unknowns $B_{j}$ by the method of algebraic least squares for $N=120$.

\begin{tabular}{lccc}
\hline$j$ & Values of $B_{j}$ & $j$ & Values of $B_{j}$ \\
\hline 1 & $-0.9014-0.0159 \mathrm{i}$ & 60 & $-4.0496 \times 10^{-6}+2.3232 \times 10^{-4} \mathrm{i}$ \\
20 & $1.8753 \times 10^{-5}-1.1013 \times 10^{-3} \mathrm{i}$ & 70 & $-5.0238 \times 10^{-6}+2.9178 \times 10^{-4} \mathrm{i}$ \\
40 & $1.8970 \times 10^{-6}-1.2080 \times 10^{-4} \mathrm{i}$ & 85 & $-3.5513 \times 10^{-6}+2.0772 \times 10^{-4} \mathrm{i}$ \\
45 & $-1.1667 \times 10^{-7}-1.8669 \times 10^{-6} \mathrm{i}$ & 120 & $2.4960 \times 10^{-6}-1.4707 \times 10^{-4} \mathrm{i}$ \\
\hline
\end{tabular}

Table 6. $R$ and $|R|$ with $K h=0.2$ and $\theta=0$, for partially immersed barrier (Type-I discretized points).

\begin{tabular}{|c|c|c|c|c|c|}
\hline \multirow{2}{*}{$a / h$} & \multicolumn{3}{|c|}{ Algebraic least squares } & \multicolumn{2}{|c|}{ Mandal and Doali } \\
\hline & $\left(\hat{m}_{1}, \hat{m}_{2}, N\right)$ & $R$ & $|R|$ & $R_{1}$ & $R_{2}$ \\
\hline \multirow[t]{3}{*}{0.2} & $(50,50,62)$ & $0.0003-0.0172 \mathrm{i}$ & 0.0173 & & \\
\hline & $(75,75,86)$ & $0.0003-0.0171 \mathrm{i}$ & 0.0171 & 0.0176 & 0.0176 \\
\hline & $(100,100,108)$ & $0.0003-0.0170 \mathrm{i}$ & 0.0170 & & \\
\hline \multirow[t]{3}{*}{0.4} & $(50,50,80)$ & $0.0053-0.0716 \mathrm{i}$ & 0.0718 & & \\
\hline & $(75,75,118)$ & $0.0052-0.0712 \mathrm{i}$ & 0.0714 & 0.0712 & 0.0713 \\
\hline & $(100,100,145)$ & $0.0051-0.0711 \mathrm{i}$ & 0.0713 & & \\
\hline \multirow[t]{3}{*}{0.6} & $(50,50,80)$ & $0.0308-0.1721 \mathrm{i}$ & 0.1748 & & \\
\hline & $(75,75,115)$ & $0.0307-0.1720 \mathrm{i}$ & 0.1747 & 0.1740 & 0.1740 \\
\hline & $(100,100,147)$ & $0.0306-0.1718 \mathrm{i}$ & 0.1745 & & \\
\hline
\end{tabular}

Table 7. $R$ and $|R|$ with $K h=0.2$ and $\theta=0$, for bottom standing barrier (Type-I discretized points).

\begin{tabular}{|c|c|c|c|c|c|}
\hline \multirow{2}{*}{$a / h$} & \multicolumn{3}{|c|}{ Algebraic least squares } & \multicolumn{2}{|c|}{ Mandal and Doali } \\
\hline & $\left(\hat{m}_{1}, \hat{m}_{2}, N\right)$ & $R$ & $|R|$ & $R_{1}$ & $R_{2}$ \\
\hline \multirow[t]{3}{*}{0.2} & $(50,50,60)$ & $0.0849-0.2765 \mathrm{i}$ & 0.2892 & & \\
\hline & $(75,75,85)$ & $0.0893-0.2839 \mathrm{i}$ & 0.2976 & 0.2914 & 0.2923 \\
\hline & $(100,100,105)$ & $0.0892-0.2840 \mathrm{i}$ & 0.2977 & & \\
\hline \multirow[t]{3}{*}{0.4} & $(50,50,80)$ & $0.0202-0.1399 \mathrm{i}$ & 0.1413 & & \\
\hline & $(75,75,115)$ & $0.0203-0.1402 \mathrm{i}$ & 0.1417 & 0.1397 & 0.1397 \\
\hline & $(100,100,140)$ & $0.0203-0.1402 \mathrm{i}$ & 0.1417 & & \\
\hline \multirow[t]{3}{*}{0.6} & $(50,50,84)$ & $0.0033-0.0569 \mathrm{i}$ & 0.0570 & & \\
\hline & $(75,75,115)$ & $0.0034-0.0574 \mathrm{i}$ & 0.0575 & 0.0573 & 0.0573 \\
\hline & $(100,100,145)$ & $0.0034-0.0574 \mathrm{i}$ & 0.0575 & & \\
\hline
\end{tabular}

Table 8. $R$ and $|R|$ with $K h=0.2$ and $\theta=0$ for partially immersed barrier (Type-II discretized points).

\begin{tabular}{|c|c|c|c|c|c|}
\hline \multirow{2}{*}{$a / h$} & \multicolumn{3}{|c|}{ Algebraic least squares } & \multicolumn{2}{|c|}{ Mandal and Doali } \\
\hline & $\left(\hat{m}_{1}, \hat{m}_{2}, N\right)$ & $R$ & $|R|$ & $R_{1}$ & $R_{2}$ \\
\hline \multirow[t]{3}{*}{0.2} & $(50,50,62)$ & $0.0003-0.0175 \mathrm{i}$ & 0.0175 & & \\
\hline & $(75,75,86)$ & $0.0003-0.0173 \mathrm{i}$ & 0.0173 & 0.0176 & 0.0176 \\
\hline & $(100,100,110)$ & $0.0003-0.0173 \mathrm{i}$ & 0.0173 & & \\
\hline \multirow[t]{3}{*}{0.4} & $(50,50,85)$ & $0.0053-0.0722 \mathrm{i}$ & 0.0724 & & \\
\hline & $(75,75,110)$ & $0.0053-0.0720 \mathrm{i}$ & 0.0720 & 0.0712 & 0.0713 \\
\hline & $(100,100,110)$ & $0.0052-0.0716 \mathrm{i}$ & 0.0718 & & \\
\hline \multirow[t]{3}{*}{0.6} & $(50,50,80)$ & $0.0314-0.1739 \mathrm{i}$ & 0.1767 & & \\
\hline & $(75,75,120)$ & $0.0309-0.1727 \mathrm{i}$ & 0.1754 & 0.1740 & 0.1740 \\
\hline & $(100,100,135)$ & $0.0308-0.1724 \mathrm{i}$ & 0.1751 & & \\
\hline
\end{tabular}


Table 9. $R$ and $|R|$ with $K h=0.2$ and $\theta=0$, for bottom standing barrier (Type-II discretized points).

\begin{tabular}{lcccr}
\hline \multirow{2}{*}{$a / h$} & \multicolumn{2}{c}{ Algebraic least squares } & & \multicolumn{2}{c}{ Mandal and Doali } \\
\cline { 2 - 5 } & $\left(\hat{m}_{1}, \hat{m}_{2}, N\right)$ & $R$ & $|R|$ & $R_{1}$ \\
\hline 0.2 & $(50,50,60)$ & $0.0895-0.2840 \mathrm{i}$ & 0.2978 & 0.2914 \\
& $(75,75,85)$ & $0.0896-0.2844 \mathrm{i}$ & 0.2981 & 0.2923 \\
0.4 & $(100,100,110)$ & $0.0894-0.2844 \mathrm{i}$ & 0.2982 & 0.1397 \\
& $(50,50,70)$ & $0.0205-0.1408 \mathrm{i}$ & 0.1423 & 0.1397 \\
0.6 & $(75,75,100)$ & $0.0204-0.1406 \mathrm{i}$ & 0.1420 & 0.0573 \\
& $(100,100,130)$ & $0.0203-0.1405 \mathrm{i}$ & 0.0579 & 0.0573 \\
& $(70,50,75)$ & $0.0034-0.0578 \mathrm{i}$ & $0.0034-0.0576 \mathrm{i}$ & \\
\hline
\end{tabular}

Table 10. $R$ and $|R|$ with $K h=0.2$ and $\theta=0$, for partially immersed barrier (Type-III discretized points).

\begin{tabular}{ccccc}
\hline \multirow{2}{*}{$a / h$} & Algebraic least squares & & Mandal and Doali \\
\cline { 2 - 5 } & $\left(\hat{m}_{1}, \hat{m}_{2}, N\right)$ & $R$ & $|R|$ & $R_{1}$ \\
\hline 0.2 & $(50,50,60)$ & $-0.0005-0.0176 \mathrm{i}$ & 0.0177 & 0.0176 \\
& $(75,75,80)$ & $-0.0002-0.0173 \mathrm{i}$ & 0.0173 & 0.0176 \\
0.4 & $(100,100,108)$ & $-0.0001-0.0172 \mathrm{i}$ & 0.0172 & 0.0712 \\
& $(50,50,85)$ & $0.0049-0.0728 \mathrm{i}$ & 0.0727 & 0.0713 \\
0.6 & $(75,75,115)$ & $0.0049-0.0715 \mathrm{i}$ & 0.0717 & 0.1740 \\
& $(100,100,145)$ & $0.0049-0.0713 \mathrm{i}$ & 0.0714 & 0.1760 \\
\end{tabular}

Table 11. $R$ and $|R|$ with $K h=0.2$ and $\theta=0$, for bottom standing barrier (Type-III discretized points).

\begin{tabular}{|c|c|c|c|c|c|}
\hline \multirow{2}{*}{$a / h$} & \multicolumn{3}{|c|}{ Algebraic least squares } & \multicolumn{2}{|c|}{ Mandal and Doali } \\
\hline & $\left(\hat{m}_{1}, \hat{m}_{2}, N\right)$ & $R$ & $|R|$ & $R_{1}$ & $R_{2}$ \\
\hline \multirow[t]{3}{*}{0.2} & $(50,50,60)$ & $0.0854-0.2773 \mathrm{i}$ & 0.2902 & & \\
\hline & $(75,75,85)$ & $0.0896-0.2843 \mathrm{i}$ & 0.2981 & 0.2914 & 0.2923 \\
\hline & $(100,100,105)$ & $0.0894 \mathrm{~d} 0.2843 \mathrm{i}$ & 0.2980 & & \\
\hline \multirow{3}{*}{0.4} & $(50,50,80)$ & $0.0203-0.1404 \mathrm{i}$ & 0.1419 & & \\
\hline & $(75,75,115)$ & $0.0203-0.1405 \mathrm{i}$ & 0.1420 & 0.1397 & 0.1397 \\
\hline & $(100,100,130)$ & $0.0203-0.1405 \mathrm{i}$ & 0.1420 & & \\
\hline \multirow[t]{3}{*}{0.6} & $(50,50,85)$ & $0.0035-0.0586 \mathrm{i}$ & 0.0587 & & \\
\hline & $(75,75,115)$ & $0.0034-0.0576 \mathrm{i}$ & 0.0577 & 0.0573 & 0.0573 \\
\hline & $(100,100,135)$ & $0.0034-0.0576 \mathrm{i}$ & 0.0577 & & \\
\hline
\end{tabular}

$0.2, a / h=0.2, \theta=0$ and $\hat{m_{1}}=\hat{m_{2}}=100$, for the case of partially immersed barrier. As expected, the error decreases as the number of unknowns $N$ increases for each case of equally and unequally discretized points. It is also found that equally spaced discretized points produce better approximation than the unequally spaced discretized points in the method applied here. The reason for this can be attributed to the fact that the functions representing the dual series relations are bounded at the end points of their intervals of validity, in the problems considered here.
Table 15 shows the computational time for different values of $N$ in the algebraic least-square method for the case of partially immersed barrier only. From this table, it is observed that the present method is computationally efficient because the present method requires a few seconds to obtain almost the same results as given in [4].

Remark

(i) The present method gives the values of the reflection coefficients in the form of complex numbers, 
Table 12. $R$ and $|R|$ with $K h=0.2$ and $\theta=0$ for partially immersed barrier (Type-IV discretized points).

\begin{tabular}{|c|c|c|c|c|c|}
\hline \multirow{2}{*}{$a / h$} & \multicolumn{3}{|c|}{ Algebraic least squares } & \multicolumn{2}{|c|}{ Mandal and Doali } \\
\hline & $\left(\hat{m}_{1}, \hat{m}_{2}, N\right)$ & $R$ & $|R|$ & $R_{1}$ & $R_{2}$ \\
\hline \multirow[t]{3}{*}{0.40 .2} & $(50,50,60)$ & $0.0003-0.0179 \mathrm{i}$ & 0.0179 & & \\
\hline & $(75,75,80)$ & $0.0003-0.0175 \mathrm{i}$ & 0.0175 & 0.0176 & 0.0176 \\
\hline & $(100,100,110)$ & $0.0003-0.0173 \mathrm{i}$ & 0.0173 & & \\
\hline \multirow[t]{3}{*}{0.40 .4} & $(50,50,85)$ & $0.0053-0.0722 \mathrm{i}$ & 0.0724 & & \\
\hline & $(75,75,110)$ & $0.0053-0.0720 \mathrm{i}$ & 0.0720 & 0.0712 & 0.0713 \\
\hline & $(100,100,110)$ & $0.0052-0.0716 \mathrm{i}$ & 0.0718 & & \\
\hline \multirow[t]{3}{*}{0.40 .6} & $(50,50,80)$ & $0.0314-0.1739 \mathrm{i}$ & 0.1767 & & \\
\hline & $(75,75,120)$ & $0.0309-0.1727 \mathrm{i}$ & 0.1754 & 0.1740 & 0.1740 \\
\hline & $(100,100,135)$ & $0.0308-0.1724 \mathrm{i}$ & 0.1751 & & \\
\hline
\end{tabular}

Table 13. $R$ and $|R|$ with $K h=0.2$ and $\theta=0$, for bottom standing barrier (Type-IV discretized points).

\begin{tabular}{ccccc}
\hline$a / h$ & & Algebraic least squares & & Mandal and Doali \\
\cline { 2 - 5 } & $\left(\hat{m}_{1}, \hat{m}_{2}, N\right)$ & $R$ & $|R|$ & $R_{1}$ \\
\hline 0.2 & $(50,50,60)$ & $0.0891-0.2834 \mathrm{i}$ & 0.2970 & 0.2914 \\
& $(75,75,75)$ & $0.0896-0.2837 \mathrm{i}$ & 0.2975 & 0.2923 \\
0.4 & $(100,100,105)$ & $0.0892-0.2840 \mathrm{i}$ & 0.2977 & 0.1397 \\
& $(50,50,82)$ & $0.0201-0.1399 \mathrm{i}$ & 0.1413 & 0.1397 \\
0.6 & $(75,75,110)$ & $0.0202-0.1402 \mathrm{i}$ & 0.1417 & 0.1417 \\
& $(100,100,120)$ & $0.0202-0.1402 \mathrm{i}$ & 0.0575 & 0.0573 \\
& $(70,50,85)$ & $0.0035-0.0574 \mathrm{i}$ & 0.0575 & 0.0573 \\
\hline
\end{tabular}

Table 14. Values of the error $E$ for different values of $N$ in the algebraic least-square method.

\begin{tabular}{lccccc}
\hline \multirow{2}{*}{$N$} & \multicolumn{5}{c}{ Error $E$} \\
\cline { 2 - 6 } & $\begin{array}{c}\text { Equally spaced } \\
\text { points }\end{array}$ & \multicolumn{4}{c}{ Unequally spaced points } \\
\cline { 2 - 6 } & Type-I & Type-II & Type-III & Type-IV \\
\hline 10 & 0.2442 & 0.3138 & 0.3113 & 0.3118 & 0.3132 \\
20 & 0.1241 & 0.1788 & 0.1746 & 0.1767 & 0.1784 \\
30 & 0.0921 & 0.1397 & 0.1373 & 0.1376 & 0.1394 \\
50 & 0.0610 & 0.0983 & 0.0960 & 0.0963 & 0.0980 \\
80 & 0.0431 & 0.0720 & 0.0699 & 0.0701 & 0.0718 \\
108 & 0.0346 & 0.0529 & 0.0517 & 0.0519 & 0.0522 \\
\hline
\end{tabular}

Table 15. Computational time for different values of $N$ in the algebraic least-square method for Case-I only, for a normal computer with RAM 4.00 GB.

\begin{tabular}{lcc}
\hline$N$ & $\begin{array}{c}\text { CPU time (s) for } \\
\text { equally spaced points }\end{array}$ & $\begin{array}{c}\text { CPU time (s) for } \\
\text { unequally spaced points }\end{array}$ \\
\hline 50 & 0.005 & 0.007 \\
70 & 0.007 & 0.067 \\
100 & 0.071 & 0.067 \\
120 & 0.023 & 0.062 \\
\hline
\end{tabular}

$R=r_{1}+\mathrm{i} r_{2}$, whereas the results presented in [4] are for the quantity $|R|$, only. It must be noted that though $|R|=\sqrt{r_{1}^{2}+r_{2}^{2}}$, knowing $R$ directly is more important for mathematical purposes.

(ii) The values of unknowns corresponding to the nonpropagating modes were not computed in [4], whereas they are investigated by the present method.

(iii) It should be remarked that the resulting integral equations in [4] finally need to be solved by reducing them to linear algebraic equations. Therefore, the presently used method of solution is direct and much simpler than the integral equation method used, by earlier researchers, for similar problems.

\section{Conclusion}

In this paper, alternative methods have been presented to solve the problems involving the scattering of water waves by thin vertical barriers in water of finite depth. The method of algebraic least-square approximation along with equal as well as unequal discretized points has been employed for the solution of the problems. Numerical values of the reflection coefficient are obtained and compared to the 
known results. It is observed that the algebraic least-square method is simpler and more straightforward, providing an effective approximation as compared with traditional integral equation approach, which is more complicated.

\section{Acknowledgements}

A Choudhary is grateful to the University Grants Commission (UGC), Government of India, for providing the research fellowship for pursuing $\mathrm{Ph} \mathrm{D}$ degree at the Indian Institute of Technology Ropar, India. S C Martha is grateful to SERB-DST, Government of India, for financial funding under Grant number SB/FTP/MS-034/2013. The authors thank Professor Aloknath Chakrabarti, Indian Institute of Science, Bangalore, for his valuable suggestions and comments during the preparation of the manuscript.

\section{Appendix: Derivation of relation (6)}

Since pressure is continuous across the gap

$$
\begin{aligned}
\left.P\right|_{x=0^{-}} & =\left.P\right|_{x=0^{+}} \\
\Rightarrow & \left.\left(\rho g y+\rho \frac{\partial \Phi}{\partial t}\right)\right|_{x=0^{-}}=\left.\left(\rho g y+\rho \frac{\partial \Phi}{\partial t}\right)\right|_{x=0^{+}} \\
& \quad(\text { using Bernoulli's equation) } \\
\Rightarrow & \left.i \sigma \phi e^{-i \sigma t}\right|_{x=0^{-}}=\left.i \sigma \phi e^{-i \sigma t}\right|_{x=0^{+}} \\
\Rightarrow & \left.\phi\right|_{x=0^{-}}=\left.\phi\right|_{x=0^{+}} .
\end{aligned}
$$

\section{References}

[1] Dean W R 1945 On the reflexion of surface waves by a submerged plane barrier. Proc. Camb Philos. Soc. 41: 231-238

[2] Ursell F 1947 The effect of a fixed barrier on surface waves in deep water. Proc. Camb Philos. Soc. 43: 374-382.

[3] Losada I J, Losada M A and Roldan A J 1992 Propagation of oblique incident waves past rigid vertical thin barriers. Appl. Ocean Res. 14: 191-199

[4] Mandal B N and Dolai D P 1994 Oblique water wave diffraction by thin vertical barriers in water of uniform finite depth. Appl. Ocean Res. 16: 195-203

[5] Mandal B N and Chakrabarti A 1999 On Galerkin's method applicable to the problems of water wave scattering by barriers. Proc. Indian Natl. Sci. Acad. 65: 61-71

[6] Sahoo T, Lee M M and Chwang A T 2000 Trapping and generation of waves by vertical porous structures. J. Eng. Mech. 126: 1074-1082

[7] Lu D Q 2004 Interaction of viscous wakes with a free surface. Appl. Math. Mech. 25(6): 647-655

[8] Xu F and Lu D Q 2011 Hydroelastic interaction between water waves and a thin elastic plate of arbitrary geometry. Sci. China-Phys. Mech. Astron. 54: 59-66

[9] Chakrabarti A and Mohapatra S 2013 Scattering of surface water waves involving semi-infinite floating elastic plates on water of finite depth. J. Marine Sci. Appl. 12: 325-333

[10] Sneddon I N 1966 Mixed boundary value problems in potential theory. Amsterdam: North-Holland 